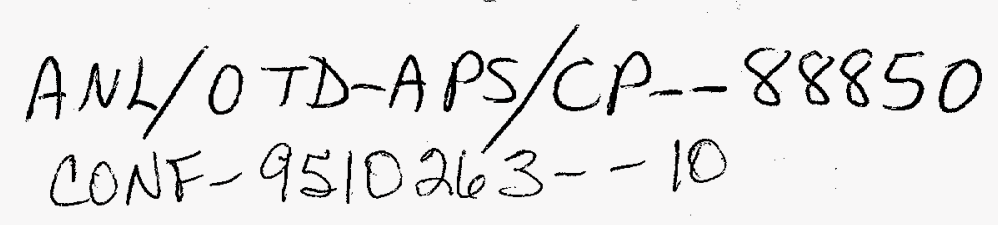

\title{
SPACE CHARGE BEAM DYNAMICS STUDIES FOR A PULSED SPALLATION SOURCE ACCELERATOR ${ }^{*}$
}

\author{
Y. Cho and E. Lessner \\ Argonne National Laboratory \\ Argonne, IL 60439 \\ U. S. A.
}

\begin{abstract}
Feasibility studies for 2-GeV, 1-MW and 10-GeV, 5-MW rapid cycling synchrotrons (RCS) for spallation neutron sources have been completed. Both synchrotrons operate at a repetition rate of $30 \mathrm{~Hz}$, and accelerate $1.04 \times 10^{14}$ protons per pulse. The injection energy of the $2-\mathrm{GeV}$ ring is $400 \mathrm{MeV}$, and the $10-\mathrm{GeV}$ RCS accepts the beam from the 2-GeV machine. Work performed to-date includes calculation of the longitudinal space charge effects in the $400-\mathrm{MeV}$ beam transfer line, and of both longitudinal and transverse space charge effects during the injection, capture and acceleration processes in the two rings. Results of space charge calculations in the rings led to proper choices of the working points and of if voltage programs that prevents beam loss. Space charge effects in the 2-GeV synchrotron, in both transverse and longitudinal phase space, have major impact on the design due to the fact that the injection energy is $400 \mathrm{MeV}$. The design achieves the required performance while alleviating harmful effects due to space charge.
\end{abstract}

\section{INTRODUCTION}

A study of the feasibility of upgrading the Intense Pulsed Neutron Source (IPNS) at ANL to a 1-MW spallation source, based on a 2-GeV RCS, has been completed [1]. An additional design study for a further upgrade from a 1-MW source to a 5-MW source, using a 10-GeV RCS, has also been completed [2]. Since transverse space charge effects are inversely proportional to $\beta^{2} \gamma^{3}$ and longitudinal space charge effects are inversely proportional to $\gamma^{2}$, space charge effects are largest at or near the $400-\mathrm{MeV}$ injection energy of the 2-GeV ring,

What follows is a brief description of accelerator configurations for the 1-MW and 5-MW proton sources, a short summary of the beam dynamics work performed to enable us to tolerate the space charge effects, and an introduction of the detailed work presented in this workshop by the other team members.

Figure 1 shows the layout of the 1-MW pulsed neutron source. It utilizes existing buildings and other infra-structure from the former 12-GeV Zero Gradient Synchrotron (ZGS). The total space being re-used for the pulsed source is about $50,000 \mathrm{~m}^{2}$. The $2-\mathrm{GeV}$ RCS is housed in the ZGS tunnel, and two neutron producing target stations are placed in former experimental area buildings. The only

\footnotetext{
* Work supported by the U. S. Department of Energy, Office of Basic Energy Sciences under the Contract W-31-109-ENG-38.
} 


\section{DISCLAMMER}

Portions of this document may be illegible in electronic image products. Images are produced from the best available original document. 
new construction required for the 1-MW facility is a shielded enclosure for the $400-\mathrm{MeV}$ linac and a building to house the linac klystrons and related equipment.

The former ZGS tunnel geometry can accommodate a 2-GeV RCS. That constraint fixes the extraction energy at $2 \mathrm{GeV}$, thus $0.5 \mathrm{~mA}$ of beam current is required to produce a 1-MW proton source. An RCS repetition rate of $30 \mathrm{~Hz}$ is preferred by the neutron source users. A $30-\mathrm{Hz}$ rate enables the users to avoid accidental events in which neutrons from previous pulses mimic those of the current pulse, referred to as frame-overlap. Low repetition rates imply that in order to get a time-averaged current of $0.5 \mathrm{~mA}$, the number of protons per pulse has to be large, in this case $1.04 \times 10^{14}$. The requirement for this large number of protons per pulse leads to space charge limit considerations.

The best injection energy to achieve $1.04 \times 10^{14}$ protons per pulse was determined to be $400-\mathrm{MeV}$ after consideration of the space charge limit dependence on $\beta^{2} \gamma^{3}$, and the ring acceptance. This will be discussed in Section III.

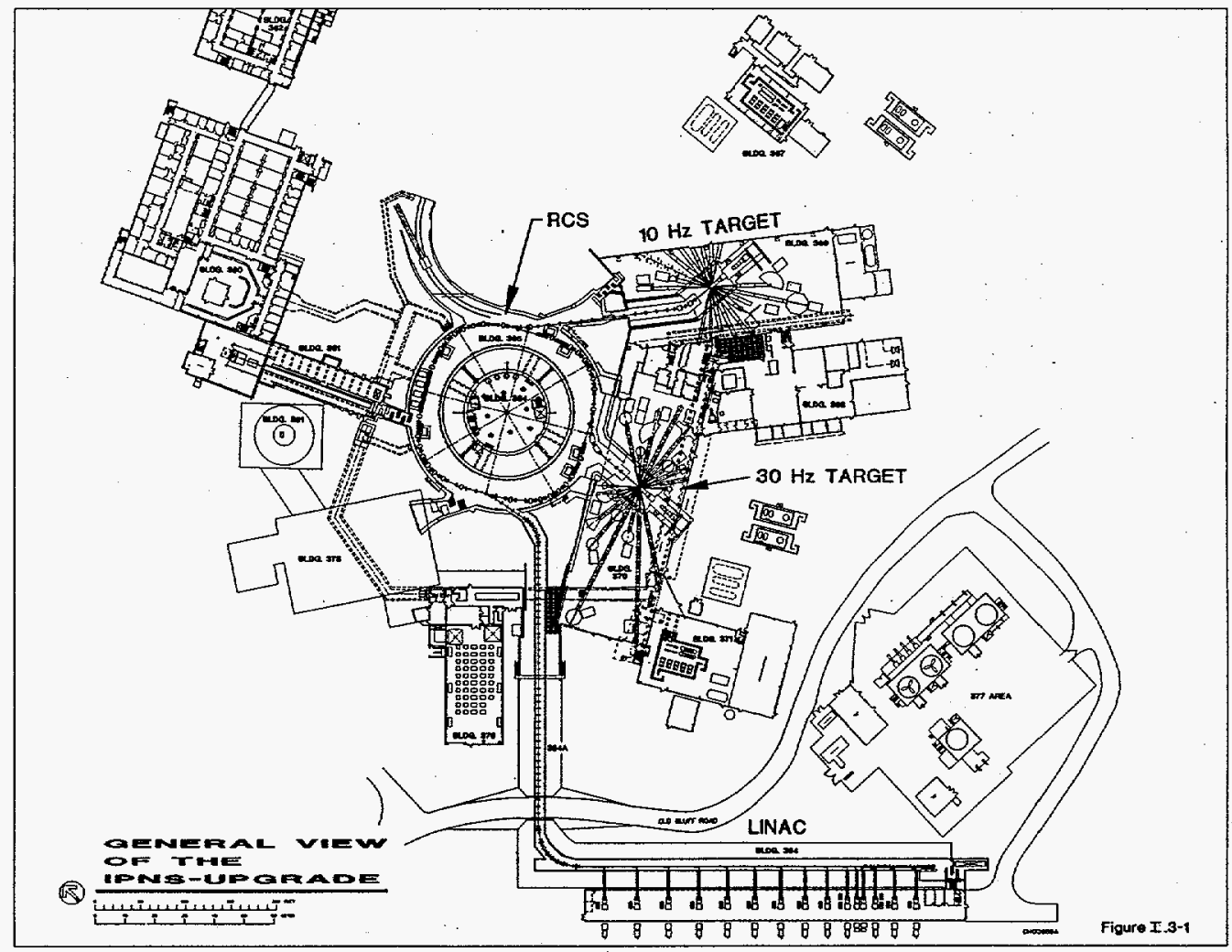

FIGURE 1. 1-MW Proton Source Machine Layout.

Figure 2 shows a general layout of the 5-MW facility based on a $10-\mathrm{GeV}$ RCS that accelerates the $2-\mathrm{GeV}(0.5-\mathrm{mA})$ beam from the $2-\mathrm{GeV}$ RCS to $10 \mathrm{GeV}$ $(0.5 \mathrm{~mA})$. In this case space charge effects are expected to be less because the 
energy of particles is higher. However, the bunch configuration in the ring is uneven, so space charge considerations should still be taken into account. The bunch configuration is uneven for geometrical reasons, as explained below.

The rf harmonic number of the $2-\mathrm{GeV}$ ring must be changed from $\mathrm{h}=1$ to $\mathrm{h}=2$ to upgrade the 1-MW facility to a $5-\mathrm{MW}$ facility, thus its $\mathrm{h}=2$ if frequency is $3 \mathrm{MHz}$. Changing the harmonic number does not change any design or performance parameters, and is done in order to raise the synchrotron frequency in the $10-\mathrm{GeV}$ ring. The circumference of the $10-\mathrm{GeV}$ RCS is 4 times that of the $2-\mathrm{GeV}$ ring. In order to further increase the synchrotron frequency of the $10-\mathrm{GeV}$ ring near its extraction energy, it is planned to inject the $2-\mathrm{GeV}$ beam into a waiting $6-\mathrm{MHz}$ bucket in the $10-\mathrm{GeV}$ ring. When two $2-\mathrm{GeV}$ beam bunches are transferred to the $10-\mathrm{GeV}$ ring, only two of 16 buckets in the large ring are occupied.

Both transverse tune shifts and if bucket distortions due to space charge must be studied during beam transfer from the low energy ring to the high energy ring.

Some of the areas in which space charge dominated beam dynamics was a consideration in designing this facility are:

1) Longitudinal space charge forces in the linac and $400-\mathrm{MeV}$ LET may alter the energy spread of the beam being injected into the 2-GeV RCS.

2) Transverse tune shift and spread may occur during injection, capture and acceleration in a $400-\mathrm{MeV}$ to $2-\mathrm{GeV}$ RCS.

3) Longitudinal space charge suppression of bucket area may occur during injection, capture and acceleration in a $400-\mathrm{MeV}$ to $2-\mathrm{GeV}$ RCS.

4) Longitudinal and transverse effects of the $2-\mathrm{GeV}$ beam in the $10-\mathrm{GeV}$ RCS must be evaluated.

The first two topics are discussed in detail in this report, and the last two topics are described in detail by E. S. Lessner elsewhere in these proceedings [3]. The possibility of injecting a Kapchinskij-Vladimirskij (KV) distribution into a circular machine is also presented in these proceedings by E. Crosbie and K. Symon [4].

\section{SPACE CHARGE EFFECTS IN THE LOW ENERGY TRANSFER LINE}

The $400-\mathrm{MeV}$ linac design included space charge effects, and the linac is described in reference [1]. The linac system consists of an $\mathrm{H}^{-}$ion source, a low energy beam transport with a chopper, a $2-\mathrm{MeV}$ radio-frequency quadrupole operating at $425 \mathrm{MHz}$, a 70-MeV drift tube linac (DTL) operating at $425 \mathrm{MHz}$, and a coupled cavity linac (CCL) operating at $1275 \mathrm{MHz}$. There is a frequency jump of a factor of three between the DTL and CCL, resulting in two empty buckets for every occupied one. 


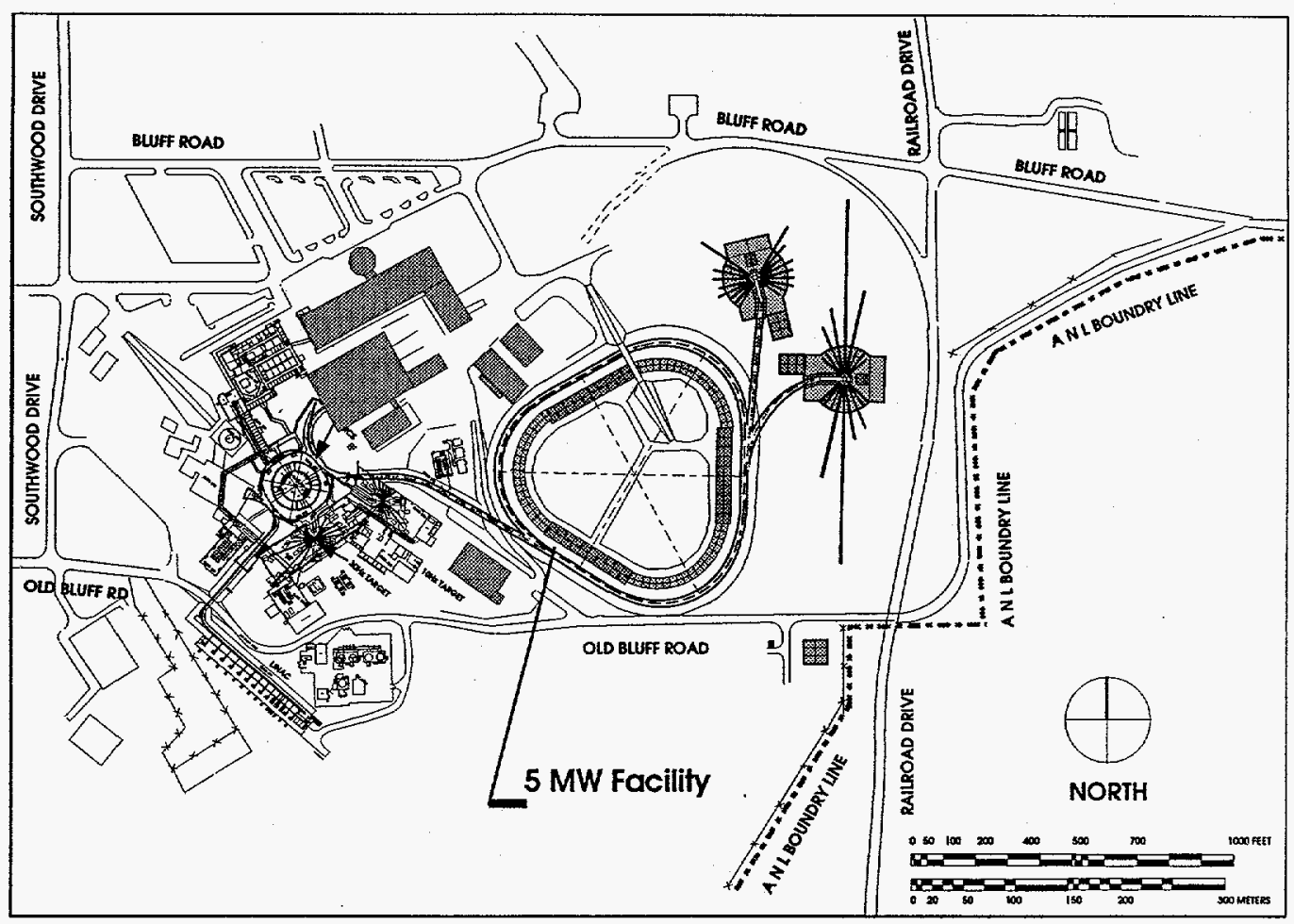

FIGURE 2. Site Layout Showing the Location of the 5-MW Facility.

The output linac micro-bunch has an energy spread of $\pm 0.8 \mathrm{MeV}$ and a phase spread of $\pm 8^{\circ}$ (in the $1275 \mathrm{MHz}$ system) measured at the base line. This corresponds to a micro-bunch phase space area of $1.4 \times 10^{-5} \mathrm{eV} \mathrm{sec}$. Each of the 0.5 -msec-long linac macro-pulses contains $1.04 \times 10^{14}$ protons. The macro-pulse is chopped at $1.1 \mathrm{MHz}$ to facilitate injection into the RCS. The chopping operation places $75 \%$ of the beam into a waiting synchrotron bucket. The remaining $25 \%$ of the beam is discarded upstream of the RFQ to avoid later losses, since it may not be captured in the RCS. These numbers can be expressed in terms of the linac pulse current. The linac average pulse current with $25 \%$ chopping is $44 \mathrm{~mA}$. The number of protons in a micro-bunch is, then, $6.3 \times 10^{8}$, and the bunch length at the base line is $\pm 17 \mathrm{psec}$ at the exit of the linac.

Longitudinal space charge effects of the $6.3 \times 10^{8}$ protons initially confined in the \pm 17 psec bucket and drifting through the low energy transport line were investigated using TRACE-3D [5], a six-dimensional tracking program that calculates the envelopes of a bunched beam while taking into account linear space charge forces.

The 157.3-m-long 400-MeV transfer line is constructed using FODO cells with the following three features: 1) a $90^{\circ}$ horizontal achromatic bend near the linac, 2) a 
3-m vertical achromatic elevation change, and 3) a $72^{\circ}$ achromatic bend near the synchrotron for injection matching.

Figure 3. shows the energy spread of the beam being transported as a function of distance from the linac exit point with and without space charge effects. It shows that when the space charge effects are taken into account, the energy spread changes very rapidly (within $20 \mathrm{~m}$ ) from the initial spread of $0.8 \mathrm{MeV}$ to a plateau of $1.6 \mathrm{MeV}$ after some $60 \mathrm{~m}$. When the space charge potential is not taken into account, the energy spread, as expected, remains constant.

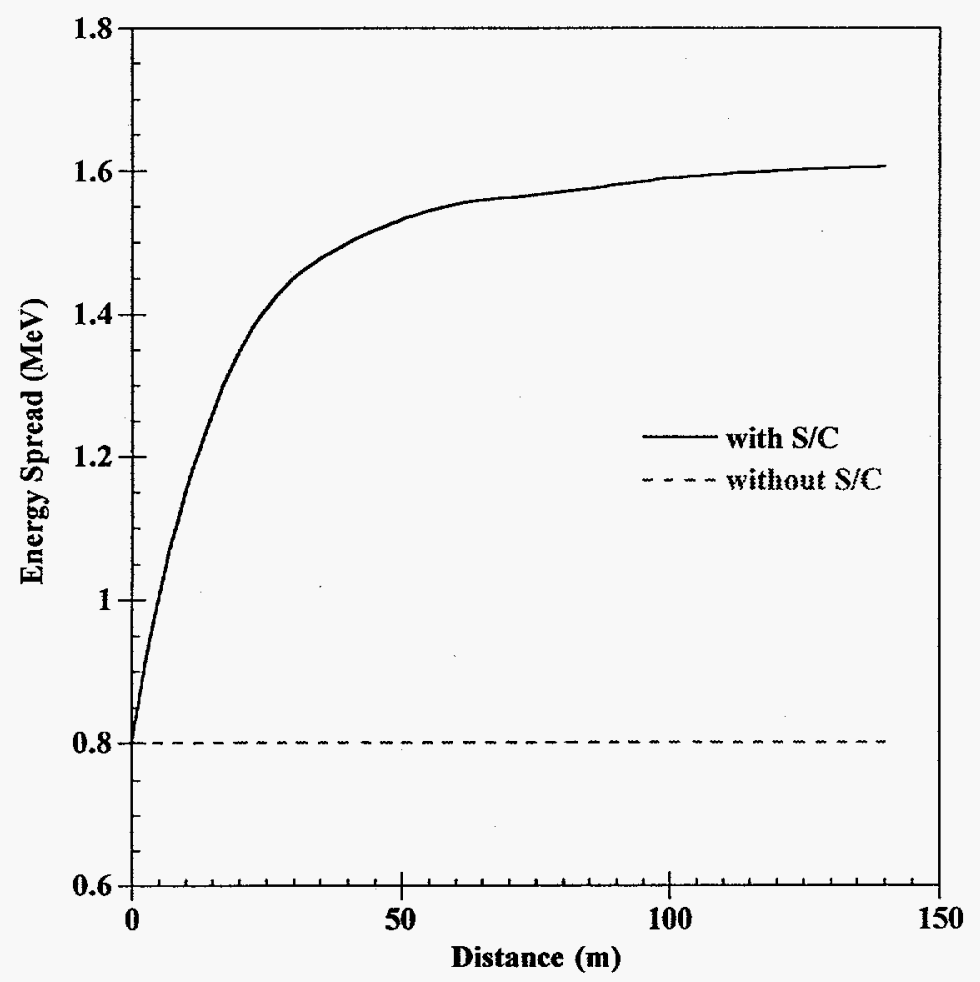

FIGURE 3. Variation of Energy Spread as a Function of Drift Distance, as Calculated with TRACE-3D.

Figure 4 shows the evolution of the micro-bunch length expressed in units of $1.275 \mathrm{GHz}$ rf phase angle. The bunch length, $\Delta \phi$, increases from $\pm 8.0^{\circ}$ to $\pm 320^{\circ}$ when space charge effects are taken into account. When space charge effects are turned off in the calculation, the bunch length increases to $\pm 150^{\circ}$. The non-linear expansion of the bunch length, particularly in the early part of the transport shown 
in the figure, comes from the fact that the head and tail of the bunch both contain particles with both $+\Delta \mathrm{E}$ and $-\Delta \mathrm{E}$ at the exit of the linac. Therefore, the time expansion of the bunch is a quadrature sum of linear expansions due to $|\Delta \mathrm{E}|$ together with travel time and the initial bunch length.

In addition to the TRACE-3D tracking study, the order of magnitude effect was estimated by analytic considerations. Calculations are further simplified by the assumption that the particles are distributed uniformly in radial space and parabolic in longitudinal space. The density is zero at the end of the bunch with the line density, $\lambda(z)$, given by:

$$
\lambda(z)=\frac{3 N}{4 z_{m}}\left(1-\frac{z^{2}}{z_{m}^{2}}\right)
$$

where, $\mathrm{N}$ is number of particles in the distribution, $\pm \mathrm{z}_{\mathrm{m}}$ is the maximum bunch length at a given point in time, and $\mathrm{z}$ is the longitudinal coordinate measured from the center of the bunch.

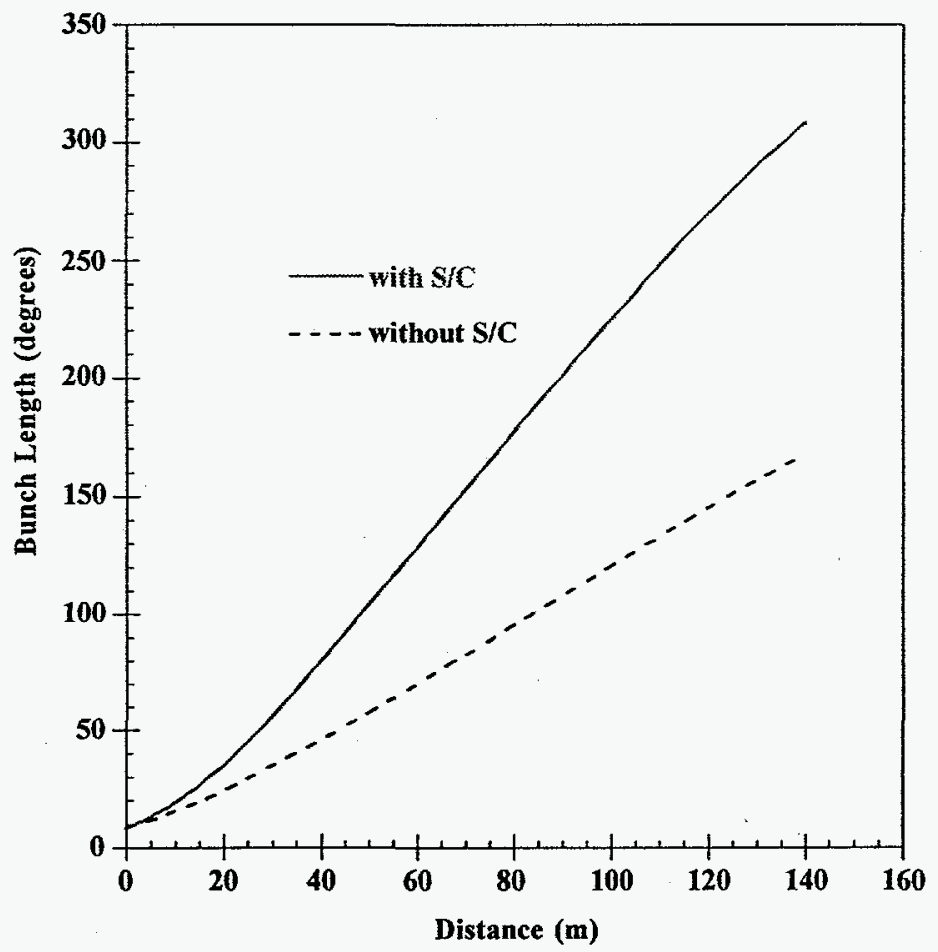

FIGURE 4. Variation of Bunch Length as a Function of Drift Distance, as Calculated with TRACE-3D. 
It is expected that during the drift, the bunch length will change due to the momentum spread in the beam, and the momentum spread of the bunch will change due to acceleration and deceleration coming from the self-field of the bunch, which varies as the bunch length changes. The coupled equations of motion for a particle in the distribution are:

$$
\begin{gathered}
\frac{d \delta z}{d t}=\frac{c \beta}{\gamma^{2}} \frac{\delta p}{p}, \\
\frac{d \delta p}{d t}=\frac{3 e^{2} N g_{o}}{8 \pi \varepsilon_{o} \gamma^{2} z_{m}^{3}} \delta z
\end{gathered}
$$

where $\delta z$ is the longitudinal position deviation and $\delta p$ is the momentum deviation from the central particle coordinates, respectively; $\varepsilon$ is the vacuum permittivity; $c, \beta$ and $\gamma$ are the speed of light and relativistic parameters; and $g_{o}=1+2 \ln (b / a)$ is a capacitive factor for a beam of radius a traversing a beampipe of radius $b$.

From the above single particle equations of motion, one can obtain the time development of the bunch envelope coordinates $\delta \mathrm{z}_{\mathrm{m}}$ and $\delta \mathrm{p}_{\mathrm{m}}$ using the WKB approximation. The envelope equations are better suited to the time development of the bunch.

$$
\begin{gathered}
\frac{d \delta z_{m}}{d t}=\frac{c \beta}{\gamma^{2} p} \delta p_{m}, \\
\frac{d \delta p_{m}}{d t}=\frac{c \beta \varepsilon^{2}}{\gamma^{2} p \delta z_{m}^{3}}+\frac{3 e^{2} N g_{o}}{8 \pi \varepsilon_{o} \gamma^{2} \delta z_{m}^{2}}
\end{gathered}
$$

where $\varepsilon$ is the longitudinal emittance of the micro-bunch $\left(1.4 \times 10^{-5} \mathrm{eV} \mathrm{sec}\right)$. Numerical calculation of the evolution of the envelope equations is performed using a leap-frog algorithm. This calculation showed that evolution of beam energy spread, $\Delta \mathrm{E}$, as the macro-pulse travels through the transport line varied from $0.8 \mathrm{MeV}$ to an asymptotic value of $1.7 \mathrm{MeV}$.

The two different computations give similar results of \pm 1.6 and $\pm 1.7 \mathrm{MeV}$. The 2-GeV RCS is designed to accept an energy spread of $\pm 2.5 \mathrm{MeV}$, and this simple analysis shows that by the time the $400-\mathrm{MeV}$ beam reaches the injection point, the energy spread has grown to $\pm 1.7 \mathrm{MeV}$. More detailed study is under way in order to confirm these preliminary conclusions. 


\section{TRANSVERSE SPACE CHARGE EFFECTS IN THE 2-GEV RCS (Space Charge Limit and the Choice of Linac Energy)}

It is customary to use the Laslett tune shift due to space charge to calculate the number of protons that can be accelerated by a synchrotron. Knowledge of the longitudinal and transverse beam distributions is required for these calculations. These distributions come either from assumptions based on previous experience or from tracking studies. The initial estimate of the space charge limit is obtained by using elliptic beam distributions and a bunching factor obtained from assumptions. A refined study of the space charge limit was performed after a longitudinal tracking study from injection to full-energy acceleration was made.

We now discuss the initial estimate of the space charge limit and choice of the injection energy to the $2-\mathrm{GeV}$ ring. The discussion presented here closely follows that described in Report of the ISIS Project Group [6]. It is reasonable to assume the local elliptical energy distribution of Hofmann and Pederson [7] for longitudinal motion of protons in the bunch. For this distribution, self-forces caused by longitudinal space charges are directly proportional to the external focusing forces. This allows bunching factors and rf bucket area reductions to be calculated analytically. The bunching factor, $B_{f}$, is defined to be the ratio of mean to peak number of particles per unit length, and is given by

$$
B_{f}=\frac{1}{2 \pi} \frac{\sin \phi_{2}-\sin \phi_{1}-\left(\phi_{2}-\phi_{1}\right) \cos \phi_{2}-0.5\left(\phi_{2}-\phi_{1}\right)^{2} \sin \phi_{s}}{\cos \phi_{s}-\cos \phi_{2}+\left(\phi_{s}-\phi_{2}\right) \sin \phi_{s}}
$$

where $\phi_{2}$ is the maximum angle, $\phi_{1}$ is the minimum angle, and $\phi_{\mathrm{s}}$ is the synchronous angle.

If we assume a small synchronous phase angle of $5^{\circ}$ for early acceleration, the bunching factor from Equation (1) is about 0.4. For the transverse charge distribution, it is reasonable to assume that the protons are distributed in an elliptical geometry of semi-axes a (horizontal) and b (vertical) with the normalized twodimensional elliptic density distribution, $\rho(x, y)$ :

$$
\rho(x, y)=\frac{3}{2 \pi a b}\left[1-\left(\frac{x}{a}\right)^{2}-\left(\frac{y}{b}\right)^{2}\right]^{1 / 2} .
$$

The density at the center of such a distribution is 1.5 times that of a beam with uniform-filled, elliptical cross section. It is believed that the density at the core of the beam soon after the injection process will not exceed the assumed elliptic distribution. The Laslett transverse space charge limit, $\mathrm{N}$, in a circular machine is directly proportional to the transverse emittance of the stacked beam and inversely 
proportional to the central density. Therefore, the space charge limit for an elliptic distribution is 1.5 times less than that of a uniformly distributed beam of similar geometry.

The Laslett incoherent transverse space charge limit can be written

where

$$
N=-\Delta v_{y} \varepsilon_{y}\left(1+\frac{a}{b}\right) \beta^{2} \gamma^{3} \frac{B_{f}}{\left(r_{p} G_{v} F_{v}\right)},
$$

$$
\begin{aligned}
& F_{v}=1+\left(1+\frac{a}{b}\right)\left(\varepsilon_{1} \frac{b^{2}}{h^{2}}+\beta^{2} \gamma^{2} B_{f}\left[\varepsilon_{1} \frac{b^{2}}{h^{2}}+\varepsilon_{2} \frac{b^{2}}{g^{2}} \frac{\rho_{m}}{R}\right]\right) / G_{v}, \\
& \mathrm{~h}=\text { vacuum chamber half-height; } \\
& \mathrm{g}=\text { bending magnet gap half-height; } \\
& \varepsilon_{1}=\text { electrostatic image coefficient }=0.1 ; \\
& \varepsilon_{2}=\text { magnetostatic image coefficient }=0.411 ; \\
& \rho_{\mathrm{m}}=\text { bending radius of the machine; } \\
& \mathrm{R}=\text { average radius of the machine; } \\
& \mathrm{r}_{\mathrm{p}}=\text { classical radius of proton }=1.5347 \times 10^{-18} \mathrm{~m} ; \\
& \Delta v_{\mathrm{y}}=\text { Allowed vertical tune shift due to space charge; } \\
& \mathrm{G}_{\mathrm{v}}=\text { form factor }=1.2 \text { for elliptic distributions and } 1.0 \text { for uniform } \\
& \varepsilon_{\mathrm{y}}=\text { distributions; and } \\
& \quad \text { the machical emittance of stacked beam at injection, or acceptance of }
\end{aligned}
$$

As Equation (2) indicates, the space charge limit is proportional to the phase space area of the stacked beam. Therefore the phase space area must be decided while taking into account its implications for such machine parameters as magnet apertures and vacuum chamber dimensions. It is prudent to iterate the values of the choices of vertical and horizontal emittances, $\varepsilon_{\mathrm{y}}$ and $\varepsilon_{\mathrm{x}}$, with consideration given to other parameters.

A detailed study led to the following conclusions:

1. Although it is customary to have $\varepsilon_{y}$ smaller than $\varepsilon_{x}$, so that the ring dipole magnet gap height can be made smaller, a very high-intensity machine like the one being described may not benefit from having $\varepsilon_{y}<\varepsilon_{x}$. The important point is to minimize the beam losses and to control them. There are some advantages to having equal acceptances, because coupling resonances can be used to stack the beam in a KV-like distribution [4]. Having equal acceptances in both planes alleviates concerns about the coupling resonances.

2. Using Equation (2) and assuming a stacked beam emittance of $375 \pi \times 10^{-6} \mathrm{~m}$ rad in each plane, the space charge limit of the machine under these conditions 
gives the number of particles per pulse as a function of the injection energy. This number can be converted to the time-averaged beam current at a repetition rate of $30 \mathrm{~Hz}$. The allowed tune shift, $\Delta v_{y}$, used is 0.15 . Figure 5 shows the average current versus the injection energy.

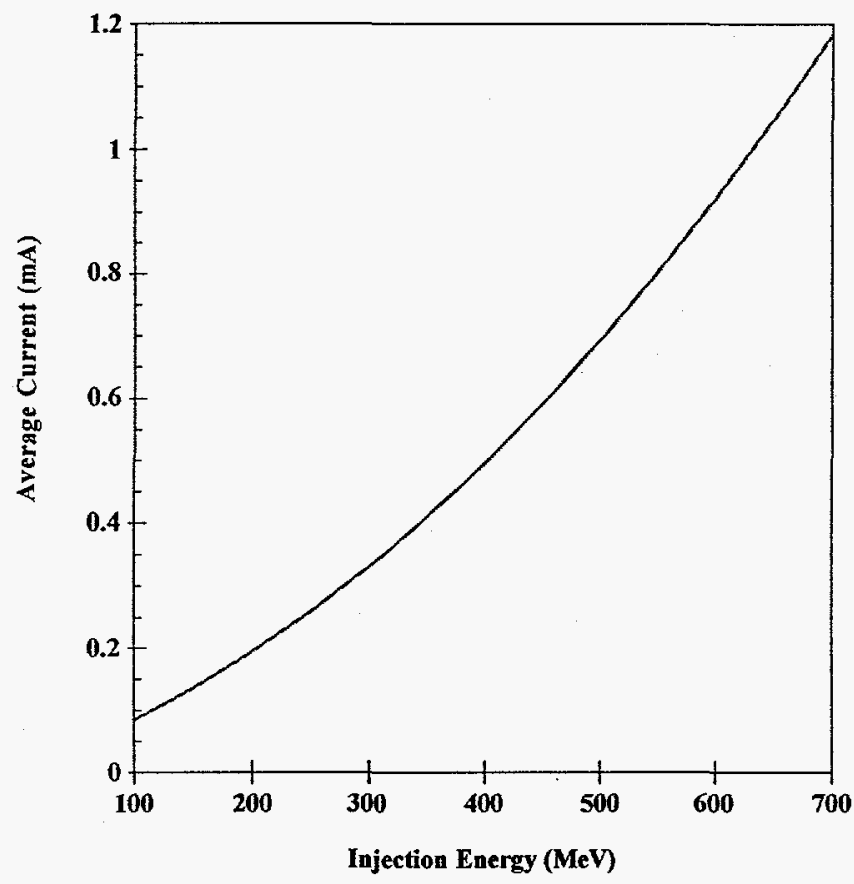

FIGURE 5. Average Current from Space Charge Limit.

$$
\Delta v_{y}=0.15, \varepsilon_{y}=\varepsilon_{x}=375 \pi \times 10^{-6} \mathrm{~m} \text {. }
$$

From this figure, one can conclude that an injector linac of $400 \mathrm{MeV}$ allows $0.5-\mathrm{mA}$ operation of the synchrotron. The current of $0.5 \mathrm{~mA}$ at a $30-\mathrm{Hz}$ repetition rate implies that the number of protons per pulse is $1.04 \times 10^{14}$.

In this preliminary estimate of the space charge limit, the use of $\Delta v_{y}=0.15$ is quite conservative, and there is a safety margin associated with the assumption. However, the calculation was based on the assumption of an elliptic charge distribution. In order to obtain refined information on the particle distribution, an extensive tracking study was performed to simulate both capture and acceleration processes and to minimize beam loss during these processes. A detailed discussion of the tracking is given in Reference [3]. The longitudinal charge distribution as a function of time from injection to extraction is obtained from the tracking study. This gives the time-varying bunching factor.

Because the goal is to accelerate $1.04 \times 10^{14}$ protons per pulse, we use the timevarying bunching factor, the emittance of $375 \times 10^{-6} \mathrm{~m}$, and Equation (2) a timevarying, incoherent, space charge tune shift. Figure 6 shows both the time-varying 
bunching factor and the space charge tune shift during the first $6 \mathrm{msec}$ of the acceleration cycle.

Note that the space charge tune shift is maximal around 1 to $2 \mathrm{msec}$ after injection, and the maximum tune shift is about 0.19 .

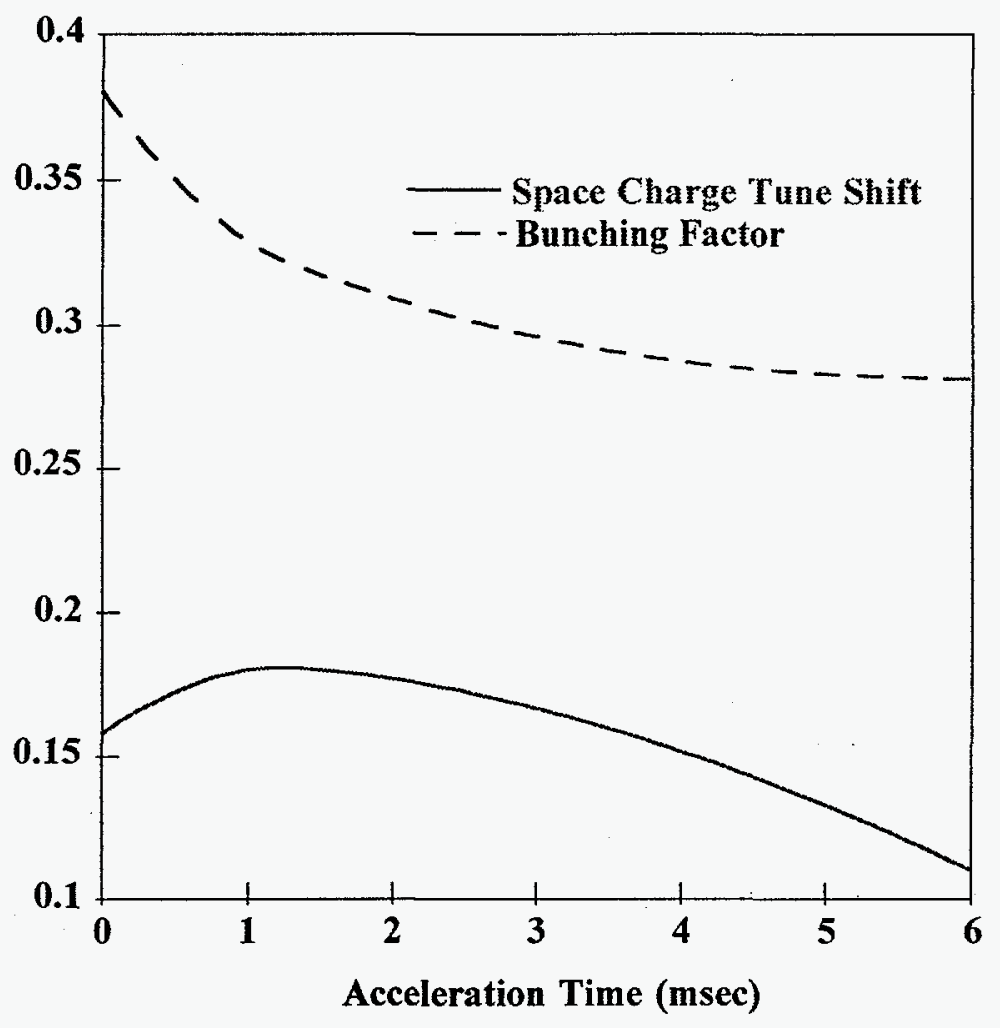

FIGURE 6. Time Variation of the Bunching Factor (top curve) and Time Variation of the Space Charge Tune Shift for the First 6 msec.

\section{LONGITUDINAL SPACE CHARGE EFFECTS IN THE 2-GEV RCS}

As expected, the longitudinal space charge effect is largest during the injection and capture processes because the effect varies as $1 / \gamma^{2}$. To study the effect in detail, we have developed an extensive longitudinal tracking study program based on a new computing code developed for this purpose [8].

The discussion on the result of the study is presented by Lessner, Cho, Harkay and Symon in these proceedings in a paper titled "Longitudinal Tracking Studies for a High Intensity Proton Synchrotron" [3]. 


\section{SPACE CHARGE EFFECTS IN THE 10-GEV RCS}

The maximum space charge effect in the $10-\mathrm{GeV}$ ring is at the injection energy of $2 \mathrm{GeV}$. The transverse Laslett tune shift at injection into 2 buckets from the $2-\mathrm{GeV}$ RCS is estimated to be 0.19 using Equation (2).

Longitudinal effects concern bucket area distortion due to space charge and matching of 2-GeV bunches from the lower energy ring to waiting buckets of the higher energy ring. Details of this work are also discussed in Reference [3].

\section{REFERENCES}

[1] "IPNS Upgrade - A Feasibility Study", ANL Report ANL-95/13 (April, 1995).

[2] Y. Cho et al., "A 10-GeV, 5-MW Proton Source for a Pulsed Spallation Source," Proc. of 13th Meeting of the International Collaborations on Advanced Neutron Sources, PSI, Villigen, Switzerland, to be published (October 11-14, 1995).

[3] E. Lessner, Y. Cho, K. Harkay and K. Symon, "Longitudinal Tracking Studies for a High Intensity Proton Synchrotron," in these proceedings.

[4] E. Crosbie and K. Symon, "Injection into a Circular Machine with a KV Distribution," in these proceedings.

[5] K. R. Crandall, "TRACE-3D Documentation," LANL Report LA-UR-90-4146, (August 1987).

[6] Report of the ISIS Project Group, Rutherford Laboratory (November 1986).

[7] A. Hofmann and F. Pederson, "Bunches with Local Elliptic Energy Distributions," IEEE Trans. Nucl. Sci. NS-26 (3), 3526 (1979).

[8] Y. Cho, E. Lessner and K. Symon, "Injection and Capture Simulations for a High Intensity Proton Synchrotron," Proc. of the European Particle Accelerator Conference, London, page 1228 (1994).

\section{DISCLAIMER}

This report was prepared as an account of work sponsored by an agency of the United States Theither the United States Government nor any agency thereof, nor any of their employees, makes any warranty, express or implied, or assumes any legal liability or responsibility for the accuracy, completeness, or usefulness of any information, apparatus, product, or process disclosed, or represents that its use would not infringe privately owned rights. Reference herein to any specific commercial product, process, or service by trade name, trademark, manufacturer, or otherwise does not necessarily constitute or imply its endorsement, recommendation, or favoring by the United States Government or any agency thereof. The views mendation, or favoring by the United States do not necessarily state or reflect those of the United States Government or any agency thereof. 